\title{
MODERATE DEVIATIONS AND ASSOCIATED LAPLACE APPROXIMATIONS FOR SUMS OF INDEPENDENT RANDOM VECTORS
}

\author{
A. DE ACOSTA
}

\begin{abstract}
Let $\left\{X_{j}\right\}$ be an i.i.d. sequence of Banach space valued r.v.'s and let $S_{n}=\sum_{j=1}^{n} X_{j}$. For certain positive sequences $b_{n} \rightarrow \infty$, we determine the exact asymptotic behavior of $E \exp \left\{\left(b_{n}^{2} / n\right) \Phi\left(S_{n} / b_{n}\right)\right\}$, where $\Phi$ is a smooth function. We also prove a large deviation principle for $\left\{\mathscr{L}\left(S_{n} / b_{n}\right)\right\}$.
\end{abstract}

\section{INTRODUCTION}

Let $\left\{X_{j}\right\}$ be a sequence of independent $E$-valued random vectors with common distribution $\mu$, where $E$ is a separable Banach space, let $S_{n}=\sum_{j=1}^{n} X_{j}$, and assume that $\left\{\mathscr{L}\left(S_{n} / n^{1 / 2}\right)\right\}$ converges weakly. Let $\left\{b_{n}\right\}$ be a positive sequence such that $b_{n} / n^{1 / 2} \rightarrow \infty$. In this paper we study certain aspects of the asymptotic behavior of $\left\{\mathscr{L}\left(S_{n} / b_{n}\right)\right\}$ (under the further assumption (1.1), $\left\{P\left\{S_{n} / b_{n} \in A\right\}\right\}$ are sometimes referred to in the literature as "probabilities of moderate deviations").

One of our results is a large deviation principle (in the sense of Varadhan [20]) for $\left\{\mathscr{L}\left(S_{n} / b_{n}\right)\right\}$ when $\left\{b_{n}\right\}$ is such that

$$
b_{n} / n \rightarrow 0 \text {. }
$$

We prove that, under appropriate integrability conditions,

$$
\begin{aligned}
& \limsup _{n \rightarrow \infty} \frac{n}{b_{n}^{2}} \log P\left\{S_{n} / b_{n} \in F\right\} \leq-\inf _{x \in F} I(x) \text { for } F \text { closed, } \\
& \liminf _{n \rightarrow \infty} \frac{n}{b_{n}^{2}} \log P\left\{S_{n} / b_{n} \in G\right\} \geq-\inf _{x \in G} I(x) \text { for } G \text { open } .
\end{aligned}
$$

The rate function $I$ depends on $\mu$ only through its covariance structure; this is in contrast to the situation that arises when $b_{n}=n$ (see [14]; also [8, 7, 2]). The precise statement is given in Theorem 2.3; Theorem 2.2 is a more general result about triangular arrays which we need in $\S 3$. Parts of Theorem 2.3 were obtained by Borovkov and Mogulskii [13] (see also de Acosta and

Received by the editors November 16, 1989.

1980 Mathematics Subject Classification (1985 Revision). Primary 60F10, 60B12.

Key words and phrases. Moderate and large deviations, exact asymptotics, Laplace approximation.

This research was partially supported by an NSF grant and by the Department of Mathematics, University of Wisconsin-Madison. 
Kuelbs [5] and Bolthausen [10]). For the case when $E$ is finite-dimensional (and the covariance matrix of $X_{1}$ is nonsingular), see Freidlin and Wentzell [17, p. 142]. Of course, in the case $E=\mathbf{R}$ there is an extensive literature on the exact asymptotics of $\left\{P\left\{S_{n} / b_{n}>x\right\}\right\}$; for classical results in this context see $[16,19]$.

If $\Phi: E \rightarrow \mathbf{R}$ is a bounded continuous function, then by Varadhan's theorem (see [15, p. 51]), (1.2) and (1.3) imply

$$
\lim _{n \rightarrow \infty} \frac{n}{b_{n}^{2}} \log E \exp \left\{\frac{b_{n}^{2}}{n} \Phi\left(\frac{S_{n}}{b_{n}}\right)\right\}=\sup _{x \in E}[\Phi(x)-I(x)] .
$$

Our main result is a refinement of (1.4), giving the exact asymptotic behavior of $E \exp \left\{\left(b_{n}^{2} / n\right) \Phi\left(S_{n} / b_{n}\right)\right\}$ when $\Phi$ is a smooth function subject to a growth condition and (1.1) is replaced by the stronger condition

$$
b_{n} / n^{2 / 3} \rightarrow 0 \text {. }
$$

Under appropriate integrability, tightness and nondegeneracy conditions, we prove that

$$
E \exp \left\{\frac{b_{n}^{2}}{n} \Phi\left(S_{n} / b_{n}\right)\right\} \sim C \exp \left\{\frac{b_{n}^{2}}{n} \sup _{x \in E}[\Phi(x)-I(x)]\right\},
$$

where the constant $C$ depends on $\Phi$ and the covariance structure of $\mu$. The precise statement is given in Theorem 3.1 and a simple example shows that condition (1.5) cannot in general be relaxed; it should be remarked that (1.5) appears also in classical results on the exact asymptotic behavior of $\left\{P\left\{S_{n} / b_{n}>x\right\}\right\}$ (see e.g. [16]). In the case $b_{n}=n$, results similar to (1.6) were obtained by Bolthausen [10] for general $E$ and previously by Martin-Löf [18] for $E=\mathbf{R}$; in contrast to (1.6), in their results the rate function $I$ is replaced by the Crámer functional of $\mu$ (see [7]). $\S 3$ of the present paper is close in spirit to Bolthausen's interesting work (he has also studied in [11] the more complicated situation that occurs in the case $b_{n}=n$ when the nondegeneracy assumption is dropped). For reference to previous results in the case when $\mu$ is Gaussian, see [10].

$\S 2$ contains the proof of the moderate deviation results and $\S 3$ that of the Laplace approximation (1.6).

Throughout the paper $E$ will denote a separable Banach space and $E^{*}$ its dual space.

\section{Moderate Deviations}

It will be convenient for the developments in $\S 3$ to prove (1.2) and (1.3) in a somewhat more general setting. Let $\left\{X_{n j}: n \in \mathbf{N}, j=1, \ldots, n\right\}$ be a triangular array of $E$-valued random vectors, each row of which is independent and identically distributed, and let $S_{n}=\sum_{j=1}^{n} X_{n j}$. We will consider the following conditions:

$$
\begin{gathered}
E X_{n 1}=0 \text { for all } n \in \mathbf{N}, \\
\left\{\mathscr{L}\left(X_{n 1}\right)\right\} \text { is tight, } \\
\sup _{n} E \exp \left(t\left\|X_{n 1}\right\|\right)<\infty \text { for all } t>0,
\end{gathered}
$$


$\left\{\mathscr{L}\left(S_{n} / n^{1 / 2}\right)\right\}$ converges weakly to a centered Gaussian measure $\gamma$,

$$
\text { If } j_{n} \in \mathbf{N}, j_{n} \rightarrow \infty, j_{n} \leq n \text {, then }\left\{\mathscr{L}\left(\sum_{j=1}^{j_{n}} X_{n j} / j_{n}^{1 / 2}\right)\right\} \text { is tight. }
$$

If $E$ is a Banach space of type 2, then it is well known that (2.4)-(2.5) follow automatically from (2.1)-(2.3); let us recall that the class of type 2 spaces includes $\mathbf{R}^{k}$, Hilbert space and $L^{p}$ spaces for $p \geq 2$ (see e.g. [6]).

Lemma 2.1. Assume that $\left\{X_{n j}\right\}$ satisfies conditions (2.1)-(2.4) and let $\left\{b_{n}\right\}$ be a positive sequence satisfying the conditions $b_{n} / n^{1 / 2} \rightarrow \infty$ and (1.1). Then

(i) There exist $c>0, n_{0} \in \mathbf{N}$ and a compact convex symmetric set $K$ such that for $n \geq n_{0}$,

$$
E \exp \left\{\frac{b_{n}}{n} q_{K}\left(S_{n}\right)\right\} \leq c^{b_{n}^{2} / n},
$$

where $q_{K}$ is the Minkowski functional of $K$.

(ii) For every $\xi \in E^{*}$,

$$
\lim _{n \rightarrow \infty} \frac{n}{b_{n}^{2}} \log E \exp \left\{\frac{b_{n}}{n}\left\langle\xi, S_{n}\right\rangle\right\}=\frac{1}{2} \int \xi^{2} d \gamma .
$$

Proof. By assumptions (2.3) and (2.4), an easy modification of Theorem 5.1(b) of [4] yields:

$$
\sup _{n} E \exp \left\{t\left\|S_{n} / n^{1 / 2}\right\|\right\}<\infty \text { for all } t>0 .
$$

Now using (2.4) and (2.6), and also (2.2) and (2.3), we obtain by Theorem 3.1 of [1]: there exists a compact, convex, symmetric set $K$ such that

$$
\sup _{n} E \exp \left\{q_{K}\left(S_{n} / n^{1 / 2}\right)\right\}<\infty, \quad \sup _{n} E \exp \left\{2 q_{K}\left(X_{n 1}\right)\right\}<\infty .
$$

We use now a well-known technique of Yurinskii [21]. For fixed $n$, let $\mathscr{F}_{0}=\{\phi, \Omega\}, \mathscr{F}_{j}=\sigma\left(X_{n 1}, \ldots, X_{n j}\right)$ for $1 \leq j \leq n$. For $j=1, \ldots, n$ let

$$
\eta_{j}=E\left[q_{K}\left(S_{n}\right) \mid \mathscr{F}_{j}\right]-E\left[q_{K}\left(S_{n}\right) \mid \mathscr{F}_{j-1}\right] .
$$

Then

$$
q_{K}\left(S_{n}\right)-E q_{K}\left(S_{n}\right)=\sum_{j=1}^{n} \eta_{j}
$$

and

$$
\left|\eta_{j}\right| \leq q_{K}\left(X_{n j}\right)+E q_{K}\left(X_{n j}\right) .
$$

Now for $0<\lambda \leq 1$,

$$
\begin{aligned}
& E \exp \left\{\lambda\left[q_{K}\left(S_{n}\right)-E q_{K}\left(S_{n}\right)\right]\right\}=E \exp \left\{\lambda \sum_{j=1}^{n} \eta_{j}\right\} \\
& =E E\left[\exp \left\{\lambda \sum_{j=1}^{n} \eta_{j}\right\} \mid \mathscr{F}_{n-1}\right]=E \exp \left\{\lambda \sum_{j=1}^{n-1} \eta_{j}\right\} E\left[\exp \left\{\lambda \eta_{n}\right\} \mid \mathscr{F}_{n-1}\right] .
\end{aligned}
$$


Next,

$$
\begin{aligned}
E\left[\exp \left[\left(\lambda \eta_{n}\right) \mid \mathscr{F}_{n-1}\right]\right. & \leq E\left[\left(1+\lambda \eta_{n}+\frac{\lambda^{2} \eta_{n}^{2}}{2} e^{\left|\eta_{n}\right|}\right) \mid \mathscr{F}_{n-1}\right] \\
& =1+\frac{\lambda^{2}}{2} E\left[\eta_{n}^{2} e^{\left|\eta_{n}\right|} \mid \mathscr{F}_{n-1}\right] \\
& \leq 1+\lambda^{2} E\left[e^{2\left|\eta_{n}\right|} \mid \mathscr{F}_{n-1}\right] \\
& \leq \exp \left\{\lambda^{2} E e^{2\left(q_{K}\left(X_{n 1}\right)+E q_{K}\left(X_{n 1}\right)\right)}\right\},
\end{aligned}
$$

using (2.8). Iterating the same procedure and taking $\lambda=b_{n} / n$ for sufficiently large $n$, we have by (2.7), (2.9) and (2.10), for certain constant $\alpha>0, \beta>0$,

$$
\begin{aligned}
E \exp \left\{\frac{b_{n}}{n} q_{K}\left(S_{n}\right)\right\} & \leq \exp \left\{\frac{b_{n}}{n} E q_{K}\left(S_{n}\right)\right\} \exp \left\{n \cdot \frac{b_{n}^{2}}{n^{2}} \alpha\right\} \\
& =\exp \left\{\frac{b_{n}}{n^{1 / 2}} E q_{K}\left(S_{n} / n^{1 / 2}\right)\right\} \exp \left\{\alpha \frac{b_{n}^{2}}{n}\right\} \\
& \leq \exp \left\{\beta \frac{b_{n}^{2}}{n}\right\} \exp \left\{\alpha \frac{b_{n}^{2}}{n}\right\}
\end{aligned}
$$

for sufficiently large $n$. This proves statement (i).

(ii) It follows from $(2.1),(2.3)$ and (2.4) that for all $\xi \in E^{*}$,

$$
\lim _{n \rightarrow \infty} E\left\langle\xi, X_{n 1}\right\rangle^{2}=\lim _{n \rightarrow \infty} E\left\langle\xi, S_{n} / n^{1 / 2}\right\rangle^{2}=\int \xi^{2} d \gamma
$$

For $\lambda \in \mathbf{R}$, we have by $(2.1)$

$$
\begin{aligned}
E \exp \left\langle\lambda \xi, S_{n}\right\rangle & =\left(E \exp \left\langle\lambda \xi, X_{n 1}\right\rangle\right)^{n} \\
& =\left(1+\frac{1}{2} \lambda^{2} E\left[\left\langle\xi, X_{n 1}\right\rangle^{2} e^{\theta \lambda\left\langle\xi, X_{n 1}\right\rangle}\right]\right)^{n}
\end{aligned}
$$

where $|\theta| \leq 1$. Setting $\lambda=b_{n} / n$, it follows by (2.3), (2.11) and dominated convergence that

$$
\lim _{n \rightarrow \infty}\left(E \exp \left\langle\frac{b_{n}}{n} \xi, S_{n}\right\rangle\right)^{n / b_{n}^{2}}=\exp \left(\frac{1}{2} \int \xi^{2} d \gamma\right) .
$$

Let us recall that the Crámer functional of $\gamma$, defined by

$$
I(x)=\sup _{\xi \in E^{*}}\left[\langle\xi, x\rangle-\frac{1}{2} \int \xi^{2} d \gamma\right],
$$

is given by

$$
I(x)= \begin{cases}\frac{1}{2}\|x\|_{\gamma}^{2}, & x \in H_{\gamma}, \\ \infty, & x \notin H_{\gamma},\end{cases}
$$

where $\left(H_{\gamma},\|\cdot\|_{\gamma}\right)$ is the Hilbert space associated to $\gamma$ (see [7]; also [12]).

We obtain now a large deviation principle for $\left\{\mathscr{L}\left(S_{n} / b_{n}\right)\right\}$.

Theorem 2.2. Let $\left\{b_{n}\right\}$ be a positive sequence satisfying the conditions $b_{n} / n^{1 / 2} \rightarrow$ $\infty$ and (1.1). Let I be given by (2.12).

(i) Assume that $\left\{X_{n j}\right\}$ satisfies (2.1)-(2.4). Then for every closed set $F$,

$$
\limsup _{n \rightarrow \infty} \frac{n}{b_{n}^{2}} \log P\left\{S_{n} / b_{n} \in F\right\} \leq-\inf _{x \in F} I(x) .
$$



$G$,

(ii) Assume that $\left\{X_{n j}\right\}$ satisfies (2.1) and (2.3)-(2.5). Then for every open set

$$
\liminf _{n \rightarrow \infty} \frac{n}{b_{n}^{2}} \log P\left\{S_{n} / b_{n} \in G\right\} \geq-\inf _{x \in G} I(x) .
$$

Proof. (i) Let $Y_{n}=b_{n} S_{n} / n, \phi(\xi)=\frac{1}{2} \int \xi^{2} d \gamma$ for $\xi \in E^{*}$. The result will follow from Theorem 2.1 of [1], taking the normalization in that result to be $b_{n}^{2} / n$ instead of $n$ (obviously the result remains valid with this change). By Lemma 2.1(ii), assumption (2.1) of [1] is verified. We show next that assumption (2.3) of [1] is satisfied. Let $a>0$, and let $K$ be as in Lemma 2.1(i). By Lemma 2.1(i), for $\alpha>0$

$$
\begin{aligned}
P\left\{\frac{n}{b_{n}^{2}} Y_{n} \notin \alpha K\right\} & =P\left\{q_{K}\left(Y_{n}\right)>\alpha \frac{b_{n}^{2}}{n}\right\} \\
& \leq e^{-\alpha \frac{b_{n}^{2}}{n}} E \exp \left\{\frac{b_{n}}{n} q_{K}\left(S_{n}\right)\right\} \\
& \leq \exp \left[-(\alpha-\log c) \frac{b_{n}^{2}}{n}\right] \quad \text { for } n \geq n_{0} \\
& \leq \exp \left[-a \frac{b_{n}^{2}}{n}\right] \text { for } n \geq n_{0}
\end{aligned}
$$

if $\alpha \geq a+\log c$.

(ii) We first remark that Lemma 3.1 of [5] is valid for triangular arrays under assumptions (2.1) and (2.3)-(2.5) (obviously the integrability assumption may be weakened). The proof is just a reinterpretation of that of Lemma 3.1 of [5]; notice that statements (3.5) and (3.6) in [5] follow from standard facts about triangular arrays; (2.5) is needed to verify (3.6) of [5] (see [3]). Now let $G$ be an open set. We must prove that if $h \in G \cap H_{\gamma}$, then

$$
\liminf _{n \rightarrow \infty} \frac{n}{b_{n}^{2}} \log P\left\{S_{n} / b_{n} \in G\right\} \geq-\frac{\|h\|_{\gamma}^{2}}{2} .
$$

But this follows from Lemma 3.2 of [5].

The following result deals with the case of an independent, identically distributed sequence. In order to formulate it, we consider the following conditions on a probability measure $\mu$ on $E$ :

$$
\begin{gathered}
\int x \mu(d x)=0, \\
\int e^{t\|x\|} \mu(d x)<\infty \text { for all } t>0, \\
\left\{\mu^{* n}\left(n^{1 / 2}(\cdot)\right)\right\} \text { is tight. }
\end{gathered}
$$

As is well known (2.15) follows from (2.13) and (2.14) if $E$ is a Banach space space of type 2 (see e.g. [6]). Under condition (2.15), $\left\{\mu^{* n}\left(n^{1 / 2}(\cdot)\right)\right\}$ converges weakly to a Gaussian measure $\gamma$ with the same covariance structure as $\mu$. Let $\left(H_{\mu},\|\cdot\|_{\mu}\right)$ be the Hilbert space associated to $\mu$; then $\left(H_{\mu},\|\cdot\|_{\mu}\right)$ depends only on the covariance structure of $\mu$ and $\left(H_{\mu},\|\cdot\|_{\mu}\right)=\left(H_{\gamma},\|\cdot\|_{\gamma}\right)$, and therefore

$$
I(x)= \begin{cases}\frac{1}{2}\|x\|_{\mu}^{2}, & x \in H_{\mu}, \\ \infty, & x \notin H_{\mu} .\end{cases}
$$


Theorem 2.3. Let $\left\{b_{n}\right\}$ be as in Theorem 2.2 and let I be given by (2.16). Let $\left\{X_{j}\right\}$ be an independent sequence with common distribution $\mu$ and let $T_{n}=$ $\sum_{j=1}^{n} X_{j}$.

(i) Assume that $\mu$ satisfies (2.13)-(2.15). Then for every closed set $F$,

$$
\limsup _{n \rightarrow \infty} \frac{n}{b_{n}^{2}} \log P\left\{T_{n} / b_{n} \in F\right\} \leq-\inf _{x \in F} I(x) .
$$

(ii) Assume that $\mu$ satisfies (2.15). Then for every open set $G$,

$$
\liminf _{n \rightarrow \infty} \frac{n}{b_{n}^{2}} \log P\left\{T_{n} / b_{n} \in G\right\} \geq-\inf _{x \in G} I(x) .
$$

Proof. The first statement is a direct corollary of Theorem 2.2(i). The second statement follows from [5], Lemmas 3.1 and 3.2.

\section{LAPLACE APPROXIMATIONS}

We have adopted in this section the framework of Bolthausen [10] for the case $b_{n}=n$. The central part of the proof of Theorem 3.1 is, however, different and is based on the decomposition given by Lemma 3.3(i); the use of this decomposition is illustrated in a simple way in the proof of Theorem 3.1(i).

In order to formulate Theorem 3.1 we will consider a probability measure $\mu$ on $E$ satisfying the following conditions; let us observe that (3.3) is a strengthening of (2.15) (take $\xi=0$ ). Let $\hat{\mu}(\xi)=\int e^{\xi} d \mu$ for $\xi \in E^{*}$.

$$
\begin{gathered}
\int x \mu(d x)=0, \\
\int e^{t\|x\|} \mu(d x)<\infty \text { for all } t>0, \\
\text { Let } \xi \in E^{*} \text { and } \alpha_{n}>0, \quad \alpha_{n} \rightarrow 0 .
\end{gathered}
$$

Let

Then

$$
d \nu_{n}=\frac{e^{\alpha_{n} \xi}}{\hat{\mu}\left(\alpha_{n} \xi\right)} d \mu, \quad v_{n}=\int x \nu_{n}(d x) .
$$

$$
\left\{\left(\nu_{n} * \delta_{-v_{n}}\right)^{* n}\left(n^{1 / 2}(\cdot)\right)\right\} \quad \text { is tight. }
$$

Condition (3.3) follows from (3.1)-(3.2) if $E$ is a type 2 space.

We will also consider a function $\Phi: E \rightarrow \mathbf{R}$ such that

$$
\begin{gathered}
\Phi \in C^{2}(E) \text { in the Fréchet sense, } \\
\Phi(x) \leq a\|x\|+b \text { for certain constants } a>0, b>0 .
\end{gathered}
$$

The following two assumptions should be considered in the light of Lemma 3.2. They also appear in [10].

(3.6) There exists a unique point $x^{*} \in E$ such that $\Phi\left(x^{*}\right)-I\left(x^{*}\right)$

$$
=\sup _{x \in E}[\Phi(x)-I(x)] \text {, where } I \text { is given by }(2.16),
$$

(3.7) For all $\xi \in E^{*}$ with $\int \xi^{2} d \mu>0$, setting $\Delta(\xi)=\int x\langle\xi, x\rangle \mu(d x)$,

$$
D^{2} \Phi\left(x^{*}\right)(\Delta(\xi), \Delta(\xi))<\int \xi^{2} d \mu .
$$


Theorem 3.1. Let $\mu$ be a probability measure on $E$ satisfying (3.1)-(3.3), let $\left\{X_{j}\right\}$ be an independent sequence with common distribution $\mu$, and let $S_{n}=$ $\sum_{j=1}^{n} X_{j}$. Let $\Phi: E \rightarrow \mathbf{R}$ satisfy (3.4) and (3.5), and assume that (3.6) and (3.7) hold.

(i) Let $\gamma$ be the Gaussian measure to which $\left\{\mu^{* n}\left(n^{1 / 2}(\cdot)\right)\right\}$ converges weakly by (3.3) with $\xi=0$. Then

$$
C=\int \exp \left\{\frac{1}{2} D^{2} \Phi\left(x^{*}\right)(y, y)\right\} \gamma(d y)<\infty .
$$

(ii) Let $\left\{b_{n}\right\}$ be a positive sequence satisfying the conditions $b_{n} / n^{1 / 2} \rightarrow \infty$ and (1.5). Then

$$
\lim _{n \rightarrow \infty} \exp \left\{-\frac{b_{n}^{2}}{n} \sup _{x \in E}[\Phi(x)-I(x)]\right\} E \exp \left\{\frac{b_{n}^{2}}{n} \Phi\left(S_{n} / b_{n}\right)\right\}=C .
$$

Remarks. (1) If, furthermore, it is assumed that $\int \xi^{3} d \mu=0$ for all $\xi \in E^{*}$, then (3.8) is valid for any positive sequence $\left\{b_{n}\right\}$ such that $b_{n} / n^{1 / 2} \rightarrow \infty$ and $b_{n} / n^{3 / 4} \rightarrow 0$. This follows from the proof of Theorem 3.1.

(2) Theorem 2.2(i) is used in the proof of Theorem 3.1(ii), but not Theorem 2.2(ii).

(3) The following example shows that assumption (1.5) cannot in general be relaxed. Let $\mu=\rho * \delta_{-1}$, where $\rho$ is the standard Poisson distribution with parameter 1 ; then $\mathscr{L}\left(S_{n} / n^{1 / 2}\right)$ converges weakly to $\gamma=N(0,1)$. Let $\Phi(x)=x$; then $\sup _{x}[\Phi(x)-I(x)]=\sup _{x}\left[x-\frac{1}{2} x^{2}\right]=\frac{1}{2}$. Also $\Phi^{\prime \prime}(x)=0$ for all $x$, so $C=1$. Now

$$
\begin{aligned}
\exp \left\{-\frac{1}{2} \frac{b_{n}^{2}}{n}\right\} E \exp \left\{\frac{b_{n}^{2}}{n} \cdot \frac{S_{n}}{b_{n}}\right\} & =\exp \left\{-\frac{1}{2} \frac{b_{n}^{2}}{n}-b_{n}+n\left(e^{b_{n} / n}-1\right)\right\}, \\
& =\exp \left\{\frac{1}{6}\left(\frac{b_{n}}{n^{2 / 3}}\right)^{3}+n O\left(\left(\frac{b_{n}}{n}\right)^{4}\right)\right\} .
\end{aligned}
$$

We shall need several lemmas for the proof of Theorem 3.1. For background information on $\left(H_{\mu},\|\cdot\|_{\mu}\right)$ we refer again to $[7,12]$.

Lemma 3.2. (i) $s=\sup _{x \in E}[\Phi(x)-I(x)]$ is attained. Moreover, if $\Phi\left(x^{*}\right)-$ $I\left(x^{*}\right)=s$, then $x^{*}=\Delta\left(D \Phi\left(x^{*}\right)\right)$; in particular, $x^{*} \in \Delta\left(E^{*}\right)$.

(ii) If $\Phi\left(x^{*}\right)-I\left(x^{*}\right)=s$, then

$$
D^{2} \Phi\left(x^{*}\right)(h, h) \leq\|h\|_{\mu}^{2} \text { for all } h \in H_{\mu} .
$$

Proof. (i) By (3.5), $\Phi(x)-I(x) \leq(a\|x\|+b)-\frac{1}{2}\|x\|_{\mu}^{2}$. Since $\|x\| \leq \sigma\|x\|_{\mu}$, where $\sigma=\sup _{\|x\|_{\mu} \leq 1}\|x\|,\left\{x \in \bar{H}_{\mu}:\|x\|_{\mu} \leq r\right\}$ is compact for all $r \geq 0$ and (3.4) holds, it follows that $s$ is finite and is attained on $H_{\mu}$.

Let $x^{*} \in H_{\mu}$ be such that $s=\Phi\left(x^{*}\right)-I\left(x^{*}\right)$. For fixed $h \in H_{\mu}, t \in \mathbf{R}$, let

$$
\begin{aligned}
f(t) & =\Phi\left(x^{*}+t h\right)-I\left(x^{*}+t h\right) \\
& =\Phi\left(x^{*}+t h\right)-\frac{1}{2}\left(\left\|x^{*}\right\|_{\mu}^{2}+2 t\left\langle x^{*}, h\right\rangle_{\mu}+t^{2}\|h\|_{\mu}^{2}\right) .
\end{aligned}
$$

Then $f^{\prime}(t)=D \Phi\left(x^{*}+t h\right)(h)-\langle x, h\rangle_{\mu}-t\|h\|_{\mu}^{2}$, and $0=f^{\prime}(0)=D \Phi\left(x^{*}\right)(h)-$ $\left\langle x^{*}, h\right\rangle_{\mu}$. It follows that for all $h \in H_{\mu}$,

$$
\left\langle\Delta\left(D \Phi\left(x^{*}\right)\right), h\right\rangle_{\mu}=D \Phi\left(x^{*}\right)(h)=\left\langle x^{*}, h\right\rangle_{\mu},
$$

implying $\Delta\left(D \Phi\left(x^{*}\right)\right)=x^{*}$. 
(ii) Similarly,

$$
0 \geq f^{\prime \prime}(0)=D^{2} \Phi\left(x^{*}\right)(h, h)-\|h\|_{\gamma}^{2} .
$$

Conditions (3.6) and (3.7) are a strengthening of the conclusions of Lemma 3.2 ; the latter one should be viewed as a nondegeneracy condition.

Lemma 3.3. (i) Let $\left\{\xi_{1}, \ldots, \xi_{k}\right\} \subset E^{*}$ be such that $\int \xi_{i} \xi_{j} d \mu=\delta_{i j}$, and let $e_{j}=\Delta\left(\xi_{j}\right)$. For $x \in E$, define

$$
P_{k}(x)=\sum_{j=1}^{k}\left\langle\xi_{j}, x\right\rangle e_{j}, \quad Q_{k}(x)=x-P_{k}(x) .
$$

Let $A$ be a continuous symmetric bilinear form on $E \times E$. Then for every $x \in E, \beta>0$,

$$
A(x, x) \leq d\left(1+\beta^{2}\right)\left\|Q_{k}(x)\right\|^{2}+\left(\alpha+\beta^{-2} d \sigma^{2}\right) \sum_{j=1}^{k}\left\langle\xi_{j}, x\right\rangle^{2},
$$

where $d=\sup \{|A(x, y)|:\|x\| \leq 1,\|y\| \leq 1\}, \alpha=\sup _{\|x\|_{\mu} \leq 1} A(x, x), \sigma=$ $\sup _{\|x\|_{\mu} \leq 1}\|x\|$.

(ii) Assume that $A$ satisfies: $A(\Delta(\xi), \Delta(\xi))<\int \xi^{2} d \mu$ for all $\xi \in E^{*}$ with $\int \xi^{2} d \mu>0$. Then $\sup _{\|x\|_{\mu} \leq 1} A(x, x)<1$.

Proof. (i) Since $x=P_{k}(x)+Q_{k}(x)$, we have

$$
A(x, x)=A\left(Q_{k}(x), Q_{k}(x)\right)+2 A\left(Q_{k}(x), P_{k}(x)\right)+A\left(P_{k}(x), P_{k}(x)\right)
$$

and therefore

$$
A(x, x) \leq d\left\|Q_{k}(x)\right\|^{2}+\alpha\left\|P_{k}(x)\right\|_{\mu}^{2}+2 d\left\|Q_{k}(x)\right\|\left\|P_{k}(x)\right\| .
$$

Now

$$
\begin{aligned}
\left\|P_{k}(x)\right\|_{\mu}^{2} & =\sum_{j=1}^{k}\left\langle\xi_{j}, x\right\rangle^{2}, \\
2\left\|Q_{k}(x)\right\|\left\|P_{k}(x)\right\| & \leq \beta^{2}\left\|Q_{k}(x)\right\|^{2}+\beta^{-2}\left\|P_{k}(x)\right\|^{2} \\
& \leq \beta^{2}\left\|Q_{k}(x)\right\|^{2}+\sigma^{2} \beta^{-2}\left\|P_{k}(x)\right\|_{\mu}^{2} .
\end{aligned}
$$

The conclusion follows from (3.9)-(3.11).

(ii) By continuity and the $\|\cdot\|_{\mu}$-density of $\Delta\left(E^{*}\right)$ in $H_{\mu}$, the assumption implies $s=\sup _{\|x\|_{\mu}=1} A(x, x) \leq 1$. Suppose $s=1$. By the compactness of $\left\{x \in H_{\mu}:\|x\|_{\mu} \leq 1\right\}$, there exists $h \in H_{\mu}$ such that $\|h\|_{\mu}=1$ and $A(h, h)=1$. Now for all $y \in H_{\mu}, t>0$,

$$
A(h+t y, h+t y) \leq\|h+t y\|_{\mu}^{2},
$$

and expanding both sides and cancelling we get

$$
2 t A(h, y)+t^{2} A(y, y) \leq 2 t\langle h, y\rangle_{\mu}+t^{2}\|y\|_{\mu}^{2} .
$$

Dividing by $t$, letting $t \rightarrow 0$ and observing that $(-y)$ may be substituted for $y$, we have $A(h, y)=\langle h, y\rangle_{\mu}$. But

$$
A(h, y)=A(h, \cdot)(y)=\langle\Delta(A(h, \cdot)), y\rangle_{\mu},
$$


and therefore for all $y \in H_{\mu}$,

$$
\langle\Delta(A(h, \cdot))-h, y\rangle_{\mu}=0,
$$

implying $h=\Delta(A(h, \cdot)) \in \Delta\left(E^{*}\right)$, which contradicts the assumption.

Lemma 3.4. Let $\left\{Y_{n j}: n \in \mathbf{N}, j=1, \ldots, n\right\}$ be a row-wise independent triangular array with

$$
d \mathscr{L}\left(Y_{n j}\right)=\frac{e^{b_{n} \varphi / n}}{\hat{\mu}\left(\frac{b_{n}}{n} \varphi\right)} d \mu \quad \text { for } j=1, \ldots, n,
$$

where $\varphi=D \Phi\left(x^{*}\right)$, and let $T_{n}=\sum_{j=1}^{n}\left(Y_{n j}-E\left(Y_{n j}\right)\right)$. Let $u_{n}=\left(n / b_{n}\right) E\left(Y_{n 1}\right)-$ $x^{*}$, and define for $v \in E$,

$$
\begin{aligned}
f_{n}(v) & =\exp \left\{\frac{b_{n}^{2}}{n}\left[\Phi\left(x^{*}+u_{n}+\frac{n^{1 / 2}}{b_{n}} v\right)-\Phi\left(x^{*}\right)-\left\langle\varphi, u_{n}+\frac{n^{1 / 2}}{b_{n}} v\right\rangle\right]\right\} \\
f(v) & =\exp \left\{\frac{1}{2} D^{2} \Phi\left(x^{*}\right)(v, v)\right\} .
\end{aligned}
$$

Then $\left\{\mathscr{L}\left(f_{n}\left(T_{n} / n^{1 / 2}\right)\right)\right\}$ converges weakly to $\gamma \circ f^{-1}$.

Proof. We first show

(i) $\lim _{n \rightarrow \infty}\left(b_{n} / n^{1 / 2}\right) u_{n}=0$.

By Lemma 3.2(i), $x^{*}=\int x\langle\varphi, x\rangle \mu(d x)$. Therefore

$$
\begin{aligned}
E\left(Y_{n 1}\right)-\frac{b_{n}}{n} x^{*}= & \frac{\int x e^{b_{n}\langle\varphi, x\rangle / n} \mu(d x)-\frac{b_{n}}{n} \int x\langle\varphi, x\rangle \mu(d x)}{\hat{\mu}\left(\frac{b_{n}}{n} \varphi\right)} \\
& +\frac{\frac{b_{n}}{n} \int x\langle\varphi, x\rangle \mu(d x)\left(1-\hat{\mu}\left(\frac{b_{n}}{n} \varphi\right)\right)}{\hat{\mu}\left(\frac{b_{n}}{n} \varphi\right)} .
\end{aligned}
$$

Now by (3.1) and the inequality $\left|e^{y}-1-y\right| \leq \frac{1}{2}|y|^{2} e^{|y|} \quad(y \in \mathbf{R})$, we have

$$
\begin{gathered}
\left\|\int x e^{b_{n}\langle\varphi, x\rangle / n} \mu(d x)-\frac{b_{n}}{n} \int x\langle\varphi, x\rangle \mu(d x)\right\| \\
=\left\|\int x\left(e^{b_{n}\langle\varphi, x\rangle / n}-1-\frac{b_{n}}{n}\langle\varphi, x\rangle\right) \mu(d x)\right\| \\
\leq \frac{1}{2} \frac{b_{n}^{2}}{n^{2}} \int\|x\|\langle\varphi, x\rangle^{2} e^{b_{n}\langle\varphi, x\rangle / n} \mu(d x) .
\end{gathered}
$$

Similarly,

$$
\left|1-\hat{\mu}\left(\frac{b_{n}}{n} \varphi\right)\right| \leq \frac{1}{2} \frac{b_{n}^{2}}{n^{2}} \int\langle\varphi, x\rangle^{2} e^{b_{n}\langle\varphi, x\rangle / n} \mu(d x) .
$$

Therefore

$$
\left\|\frac{b_{n}}{n^{1 / 2}} u_{n}\right\|=\left\|n^{1 / 2}\left(E\left(Y_{n 1}\right)-\frac{b_{n}}{n} x^{*}\right)\right\| \leq n^{1 / 2} \frac{b_{n}^{2}}{n^{2}} r_{n},
$$

with $\left\{r_{n}\right\}$ bounded. Since $n^{1 / 2} b_{n}^{2} / n^{2}=\left(b_{n} / n^{3 / 4}\right)^{2}$, claim (i) follows by assumption (1.5). 
We prove next

(ii) For every $r>0, \lim _{n \rightarrow \infty} \sup _{\|v\| \leq r}\left|f_{n}(v)-f(v)\right|=0$.

In fact, by Taylor's formula,

$\left|\log f_{n}(v)-\log f(v)\right|$

$$
\begin{aligned}
& =\frac{1}{2} \mid D^{2} \Phi\left(x^{*}+\theta\left(u_{n}+\frac{n^{1 / 2}}{b_{n}} v\right)\right)\left(\frac{b_{n}}{n^{1 / 2}} u_{n}+v, \frac{b_{n}}{n^{1 / 2}} u_{n}+v\right) \\
& \leq \frac{1}{2}\left\|D^{2} \Phi\left(x^{*}+\theta\left(u_{n}+\frac{n^{1 / 2}}{b_{n}} v\right)\right)-D^{2} \Phi\left(x^{*}\right)(v, v)|| \mid \frac{b_{n}}{n^{1 / 2}} u_{n}+v\right\|^{2} \\
& +\frac{1}{2}\left|D^{2} \Phi\left(x^{*}\right)\left(\frac{b_{n}}{n^{1 / 2}} u_{n}+v, \frac{b_{n}}{n^{1 / 2}} u_{n}+v\right)-D^{2} \Phi\left(x^{*}\right)(v, v)\right|,
\end{aligned}
$$

where $|\theta| \leq 1$, and claim (ii) follows by assumption (3.4) and claim (i).

By [9, p. 34] and claim (ii), the proof will be completed if we show that

$$
\left\{\mathscr{L}\left(T_{n} / n^{1 / 2}\right)\right\} \text { converges weakly to } \gamma \text {. }
$$

By assumption (3.3), $\left\{\mathscr{L}\left(T_{n} / n^{1 / 2}\right)\right\}$ is tight. Therefore it is enough to show that $\left\{\mathscr{L}\left(\left\langle\xi, T_{n} / n^{1 / 2}\right\rangle\right)\right\}$ converges weakly to $\gamma \circ \xi^{-1}$ for $\xi \in E^{*}$. But it is easily shown that

$$
\begin{gathered}
E\left\langle\xi, T_{n} / n^{1 / 2}\right\rangle^{2} \rightarrow \int \xi^{2} d \gamma, \\
\lim _{n \rightarrow \infty} E\left(\left\langle\xi, Y_{n 1}-E\left(Y_{n 1}\right)\right\rangle^{2} I_{\left\{\left|\left\langle\xi, Y_{n 1}-E\left(Y_{n 1}\right)\right\rangle\right|>n^{1 / 2} \varepsilon\right\}}\right)=0
\end{gathered}
$$

for every $\varepsilon>0$, so (3.11) follows by Lindeberg's theorem (for triangular arrays).

Lemma 3.5. Let $\left\{U_{n j}: j=1, \ldots, n ; n \in \mathbf{N}\right\}$ be a triangular array of $E$-valued random vectors such that each row is independent and identically distributed. Let $V_{n}=\sum_{j=1}^{n} U_{n j}$, and let $q$ be a continuous seminorm on $E$. Assume

(1) For all $n, E\left(U_{n 1}\right)=0$,

(2) For all $t>0, \sup _{n} E \exp \left\{t q\left(U_{n 1}\right)\right\}<\infty$,

(3) $\sup _{n} E q\left(V_{n} / n^{1 / 2}\right)<\infty$.

Let $a=\inf _{h \in \mathbf{N}} \limsup \sup _{n \rightarrow \infty}\left(E q^{2}\left(h^{-1 / 2} \sum_{j=1}^{h} U_{n j}\right)\right)^{1 / 2}$. Then for all $\rho<$ $\left(8 a^{2}\right)^{-1}$ and sufficiently small $\delta>0$,

$$
\sup _{n} E\left(\exp \left\{\rho q^{2}\left(V_{n} / n^{1 / 2}\right)\right\} I_{\left\{q\left(V_{n}\right)<\delta n\right\}}\right)<\infty
$$

Proof. Let $b>a$. Choose $h, n_{0} \in \mathbf{N}$ such that for $n \geq n_{0}$,

$$
E q^{2}\left(h^{-1 / 2} \sum_{j=1}^{h} U_{n j}\right)<b^{2}
$$

Let $j_{n}=\left[\frac{n}{h}\right]$ and for $n \geq h, i=1, \ldots, j_{n}$, define

$$
Z_{n i}=h^{-1 / 2} \sum_{j=(i-1) h+1}^{i h} U_{n j}, \quad W_{j_{n}}=\sum_{i=1}^{j_{n}} Z_{n i} .
$$


The triangular array $\left\{Z_{n i}: n \geq h, i=1, \ldots, j_{n}\right\}$ has independent, identically distributed rows. Next, for all $n \geq h, m \in \mathbf{N}, m>2$,

$$
\begin{aligned}
E q^{m}\left(Z_{n 1}\right) & =\int_{0}^{\infty} m t^{m-1} P\left\{q\left(Z_{n 1}\right)>t\right\} d t \\
& \leq \int_{0}^{\infty} m t^{m-1} e^{-t} E \exp \left\{q\left(Z_{n 1}\right)\right\} d t \\
& \leq m ! L,
\end{aligned}
$$

where $L=\sup _{n \geq h} E \exp \left\{q\left(Z_{n 1}\right)\right\}<\infty$ by assumption (2). By (3.12) and (3.13), we have for all $n \geq \max \left\{h, n_{0}\right\}, m \geq 2$,

$$
E q^{m}\left(Z_{n 1}\right) \leq\left(\frac{m !}{2}\right) b^{2} H^{m-2}
$$

where $H=\max \left\{2 b^{-2} L, 1\right\}$.

By (3.14), applying to the $n$th row of $\left\{Z_{n i}\right\}$ Theorem 2.1 of Yurinskii [21], with $b_{j}^{2}=b^{2}$ (so $\left.B_{n}^{2}=j_{n} b^{2}\right), \beta_{n}=E q\left(W_{j_{n}}\right)$, we have: for $s>0$, setting $c_{n}=E q\left(W_{j_{n}} / j_{n}^{1 / 2}\right)$,

$$
\begin{aligned}
P\left\{q\left(W_{j_{n}}\right)>s j_{n}^{1 / 2}\right\} & =P\left\{q\left(W_{j_{n}}\right)>\left(\frac{s}{b}\right) b j_{n}^{1 / 2}\right\} \\
& \leq \exp \left\{-\frac{1}{8 b^{2}}\left(s-c_{n}\right)^{2}\left(1+\frac{\left(s-c_{n}\right) H}{2 b^{2} j_{n}^{1 / 2}}\right)^{-1}\right\} .
\end{aligned}
$$

Now for $0<\lambda<1, n$ sufficiently large, $t>0$

$$
\begin{aligned}
P\left\{q\left(\frac{V_{n}}{n^{1 / 2}}\right)>t\right\} \leq & P\left\{q\left(h^{1 / 2} W_{j_{n}}\right)>\lambda t_{n}^{1 / 2}\right\} \\
& +P\left\{q\left(V_{n}-h^{1 / 2} W_{j_{n}}\right)>(1-\lambda) t n^{1 / 2}\right\} \\
\leq & P\left\{q\left(W_{j_{n}}\right)>\lambda t j_{n}^{1 / 2}\right\} \\
& +\sup _{l \leq h} P\left\{q\left(\sum_{j=1}^{l} U_{n j}\right)>(1-\lambda) t n^{1 / 2}\right\} .
\end{aligned}
$$

For $\tau>0, l \leq h, n$ sufficiently large, we have

$$
P\left\{q\left(\sum_{j=1}^{l} U_{n j}\right)>(1-\lambda) t n^{1 / 2}\right\} \leq \exp \left\{-\tau t n^{1 / 2}\right\} M,
$$

where $M=\sup _{n}\left(E \exp \left\{\tau(1-\lambda)^{-1} q\left(U_{n 1}\right)\right\}\right)^{h}<\infty$ by assumption (2).

By (3.15)-(3.17) we have for $t>0,0<\lambda<1$ and $n$ sufficiently large,

$$
\begin{aligned}
P\left\{q\left(\frac{V_{n}}{n^{1 / 2}}\right)>t\right\} \leq & \exp \left\{-\frac{1}{8 b^{2}}\left(\lambda t-c_{n}\right)^{2}\left(1+\frac{\left(\lambda t-c_{n}\right) H}{2 b^{2} j_{n}^{1 / 2}}\right)^{-1}\right\} \\
& +M \exp \left\{-\tau t n^{1 / 2}\right\} .
\end{aligned}
$$

Using assumption (1), it is easily seen that

$$
\limsup _{n \rightarrow \infty} c_{n} \leq \limsup _{n \rightarrow \infty} E q\left(\frac{V_{n}}{n^{1 / 2}}\right)=c, \quad \text { say. }
$$


For $\frac{2}{3}<\lambda<1$ and sufficiently large $n$, we have by (3.18) and (3.19):

$$
\begin{aligned}
E(\exp & \left.\left\{\rho q^{2}\left(\frac{V_{n}}{n^{1 / 2}}\right)\right\} I_{\left\{q\left(V_{n}\right)<\delta n\right\}}\right) \\
= & 1+\int_{0}^{\delta n^{1 / 2}} 2 \rho t e^{\rho t^{2}} P\left\{q\left(\frac{V_{n}}{n^{1 / 2}}\right)>t\right\} d t \\
\leq & 1+\int_{0}^{3 c} \cdots+\int_{3 c}^{\delta n^{1 / 2}} 2 \rho t e^{\rho t^{2}} \\
& \times \exp \left\{-\frac{1}{8 b^{2}}(\lambda t-2 c)^{2}\left(1+\frac{\delta \lambda H(2 h)^{1 / 2}}{2 b^{2}}\right)^{-1}\right\} d t \\
& +\int_{3 c}^{\delta n^{1 / 2}} 2 \rho t e^{\rho \delta n^{1 / 2} t} M \exp \left\{-\tau t n^{1 / 2}\right\} d t .
\end{aligned}
$$

It is now clear that if $\rho<\left(8 a^{2}\right)^{-1}$, by taking $b$ sufficiently close to $a, \lambda$ sufficiently close to $1, \delta$ sufficiently small and $\tau$ sufficiently large, the statement follows.

Lemma 3.6. Let $\left\{U_{n j}: j=1, \ldots, n \in \mathbf{N}\right\}$ be a triangular array of $E$-valued random vectors such that each row is independent and identically distributed, and let $V_{n}=\sum_{j=1}^{n} U_{n j}$. Assume

(1) For all $n, E\left(U_{n 1}\right)=0$,

(2) For every bounded finite dimensional set $A \subset E^{*}$,

$$
\sup _{n} \sup _{\xi \in A} E \exp \left\{\left|\left\langle\xi, U_{n 1}\right\rangle\right|\right\}<\infty \text {. }
$$

(3) $\left\{\mathscr{L}\left(U_{n 1}\right)\right\}$ converges weakly to $\mu$.

Let $\left\{\xi_{j}: j \in \mathbf{N}\right\}$ be such that $\int \xi_{i} \xi_{j} d \mu=\delta_{i j}$. Then for every $\eta<1$ and every $k \in \mathbf{N}$, there exists $\delta>0$ such that

$$
\sup _{n} E\left(\exp \left\{\frac{1}{2} \eta^{2} \sum_{j=1}^{k}\left\langle\xi_{j}, \frac{V_{n}}{n^{1 / 2}}\right\rangle^{2}\right\} I_{\left\{\left\|V_{n}\right\|<\delta n\right\}}\right)<\infty .
$$

Proof. Fix $\eta<1$ and $k \in \mathbf{N}$. Since $\left|\left\langle\xi_{j}, V_{n}\right\rangle\right| \leq\left\|\xi_{j}\right\|\left\|V_{n}\right\|$ for $j=1, \ldots, k$, it is enough to prove: for sufficiently small $\delta>0$,

$$
\sup _{n} E\left(\exp \left\{\frac{1}{2} \eta^{2} \sum_{j=1}^{k}\left\langle\xi_{j}, \frac{V_{n}}{n^{1 / 2}}\right\rangle^{2}\right\} I_{\left\{\left|\left\langle\xi_{j}, V_{n}\right\rangle\right|<\delta n, j=1, \ldots, k\right\}}\right)<\infty .
$$

Let $\lambda$ be the canonical Gaussian measure in $\mathbf{R}^{k}$. Then

$$
\begin{aligned}
& E\left(\exp \left\{\frac{1}{2} \eta^{2} \sum_{j=1}^{k}\left\langle\xi_{j}, \frac{V_{n}}{n^{1 / 2}}\right\rangle^{2}\right\} I_{\left\{\left|\left\langle\xi_{j}, V_{n}\right\rangle\right|<\delta n, j=1, \ldots, k\right\}}\right) \\
& =E\left(\left[\int \exp \left\{\eta \sum_{j=1}^{k} z_{j}\left\langle\xi_{j}, \frac{V_{n}}{n^{1 / 2}}\right\rangle\right\} \lambda(d z)\right] I_{\left\{\left|\left\langle\xi_{j}, V_{n}\right\rangle\right|<\delta n, j=1, \ldots, k\right\}}\right) \\
& =\int \lambda(d z) E\left(\exp \left\{\eta \sum_{j=1}^{k} z_{j}\left\langle\xi_{j}, \frac{V_{n}}{n^{1 / 2}}\right\rangle\right\} I_{\left\{\left|\left\langle\xi_{j}, V_{n}\right\rangle\right|<\delta n, j=1, \ldots, k\right\}}\right),
\end{aligned}
$$

where $z=\left(z_{1}, \ldots, z_{k}\right) \in \mathbf{R}^{k}$. 
For $i=1, \ldots, n, z \in \mathbf{R}^{k}$, let $Y_{n i}^{(z)}=\sum_{j=1}^{k} z_{j}\left\langle\xi_{j}, U_{n i}\right\rangle$, and let $W_{n}^{(z)}=$ $\sum_{i=1}^{n} Y_{n i}^{(z)}$. Since, setting $|z|^{2}=\sum_{j=1}^{k} z_{j}^{2}$, we have

$$
\left\{\left|\left\langle\xi_{j}, V_{n}\right\rangle\right|<\delta n, j=1, \ldots, k\right\} \subset\left\{\left|W_{n}^{(z)}\right|<\delta|z| k^{1 / 2} n\right\},
$$

by (3.21) in order to prove (3.20) it is enough to show: for sufficiently small $\delta>0$,

$$
\sup _{n} \int \lambda(d z) E\left(\exp \left\{\eta \frac{W_{n}^{(z)}}{n^{1 / 2}}\right\} I_{\left\{\left|W_{n}^{(z)}\right|<\delta|z| n\right\}}\right)<\infty .
$$

In order to prove (3.22) we estimate $P\left\{\left|W_{n}^{(z)} / n^{1 / 2}\right|>t\right\}$ by means of Bernstein's inequality (see [21, p. 474], or [19, p. 55]). We have $E\left(Y_{n 1}^{(z)}\right)=0$ for $n \in \mathbf{N}$ by assumption (1). Also

$$
\begin{aligned}
E\left(Y_{n 1}^{(z)}\right)^{2}-\sum_{j=1}^{k} z_{j}^{2} & =\sum_{j} \sum_{l} z_{j} z_{l}\left[E\left(\left\langle\xi_{j}, U_{n 1}\right\rangle\left\langle\xi_{l}, U_{n 1}\right\rangle\right)-\int \xi_{j} \xi_{l} d \mu\right] \\
& \leq \sup _{j, l}\left|E\left(\left\langle\xi_{j}, U_{n 1}\right\rangle\left\langle\xi_{l}, U_{n 1}\right\rangle\right)-\int \xi_{j} \xi_{l} d \mu\right|\left(\sum_{j}\left|z_{j}\right|\right)^{2} \\
& \leq \sup _{j, l}\left|E\left(\left\langle\xi_{j}, U_{n 1}\right\rangle\left\langle\xi_{l}, U_{n 1}\right\rangle\right)-\int \xi_{j} \xi_{l} d \mu\right| k\left(\sum_{j} z_{j}^{2}\right)
\end{aligned}
$$

and therefore by assumptions (2) and (3), given $\zeta>1$ (to be further specified later), there exists $n_{0}$ such that for $n \geq n_{0}$,

$$
E\left(Y_{n 1}^{(z)}\right)^{2} \leq \zeta|z|^{2}
$$

Next,

$$
\begin{aligned}
P\left\{\left|Y_{n 1}^{(z)}\right|>t\right\} & \leq \exp \left\{-\frac{t}{|z|}\right\} E \exp \left\{\frac{1}{|z|}\left|Y_{n 1}^{(z)}\right|\right\} \\
& \leq a \exp \left\{-\frac{t}{|z|}\right\},
\end{aligned}
$$

where $a=\sup _{n} \sup _{|w| \leq 1} E \exp \left\{\left|Y_{n 1}^{(w)}\right|\right\}<\infty$ by assumption (2). Therefore, for $m>2, n \in \mathbf{N}$,

$$
\begin{aligned}
E\left|Y_{n 1}^{(z)}\right|^{m} & =\int_{0}^{\infty} m t^{m-1} P\left\{\left|Y_{n 1}^{(z)}\right|>t\right\} d t \\
& \leq \int_{0}^{\infty} m t^{m-1} a \exp \left\{-\frac{t}{|z|}\right\} d t \\
& =a m !|z|^{m} \leq\left(\frac{m !}{2}\right) \zeta|z|^{2}\left(2 a \zeta^{-1}|z|\right)^{m-2},
\end{aligned}
$$

since we may assume $2 a \zeta^{-1} \geq 1$. By (3.23), (3.24) and Bernstein's inequality, for $n \geq n_{0}$ and $t>0$,

$$
P\left\{\left|\frac{W_{n}^{(z)}}{n^{1 / 2}}\right|>t\right\} \leq 2 \exp \left\{-\frac{t^{2}}{2 \zeta|z|^{2}}\left(1+\frac{2 a t}{n^{1 / 2} \zeta^{2}|z|}\right)^{-1}\right\} .
$$


Now by (3.25), for sufficiently large $n$,

$$
\begin{aligned}
& E\left(\exp \left\{\eta\left|\frac{W_{n}^{(z)}}{n^{1 / 2}}\right|\right\} I_{\left\{\left|W_{n}^{(z)} / n^{1 / 2}\right|<\delta|z| n^{1 / 2}\right\}}\right) \\
& \quad=1+\int_{0}^{\delta|z| n^{1 / 2}} \eta \exp (\eta t) P\left\{\left|\frac{W_{n}^{(z)}}{n^{1 / 2}}\right|>t\right\} d t \\
& \leq 1+\int_{0}^{\delta|z| n^{1 / 2}} \eta \exp (\eta t) \cdot 2 \exp \left\{-\frac{t^{2}}{2 \zeta|z|^{2}}\left(1+\frac{2 a t}{n^{1 / 2} \zeta^{2}|z|}\right)^{-1}\right\} d t \\
& \leq 1+2 \int_{0}^{\infty} \eta \exp (\eta t) \exp \left\{-\frac{t^{2}}{2 \zeta|z|^{2}}\left(1+2 a \zeta^{-2} \delta\right)^{-1}\right\} d t \\
& \leq 1+2 \sqrt{2 \pi} \eta \alpha|z| \exp \left\{\frac{1}{2}(\eta \alpha)^{2}|z|^{2}\right\}
\end{aligned}
$$

by an elementary computation, where $\alpha=\zeta^{1 / 2}\left(1+2 a \zeta^{-2} \delta\right)^{1 / 2}$. Choosing $\zeta>1$ and $\delta>0$ so that $\eta \alpha<1$, the result follows from (3.22).

Proof of Theorem 3.1. (i) The fact that $C<\infty$ follows from the integrability arguments in the proof of (ii). Nevertheless, we will give a simple direct proof because it illustrates the use of the decomposition given by lemma 3.3(i), which is later employed in a more complicated situation in the proof of (ii).

Let $\left\{\xi_{j}: j \in \mathbf{N}\right\} \subset E^{*}$ be such that $\left\{\Delta\left(\xi_{j}\right): j \in \mathbf{N}\right\}$ is an orthonormal basis of $H_{\gamma}=H_{\mu}$ (see [12]). Let $A=D^{2} \Phi\left(x^{*}\right)$. By (3.7) and Lemma 3.3(ii), $\alpha=\sup _{\|x\|_{\gamma} \leq 1} A(x, x)<1$. Choose $\beta>0$ such that $\rho=\alpha+\beta^{-2} d \sigma^{2}<1$. Since $Q_{k}(Z) \rightarrow 0$ a.s. for a random vector $Z$ with $\mathscr{L}(Z)=\gamma$ (see [12]), one may choose $k \in \mathbf{N}$ such that

$$
\int \exp \left(\frac{1}{2} d\left(1+\beta^{2}\right)\left\|Q_{k}(x)\right\|^{2}\right) \gamma(d x)<\infty .
$$

By Lemma 3.3(i)

$$
\begin{aligned}
\int \exp & \left\{\frac{1}{2} A(y, y)\right\} \gamma(d y) \\
\leq & \int \exp \left\{\frac{1}{2} d\left(1+\beta^{2}\right)\left\|Q_{k}(x)\right\|^{2}\right\} \gamma(d x) \\
& \times \int \exp \left\{\frac{1}{2} \rho \sum_{j=1}^{k}\left\langle\xi_{j}, x\right\rangle^{2}\right\} \gamma(d x)<\infty
\end{aligned}
$$

since the second integral on the right-hand side is

$$
\int_{\mathbf{R}^{k}} \exp \left\{\frac{1}{2} \rho|z|^{2}\right\} \lambda(d z)<\infty
$$

because $\rho<1$, where $|\cdot|$ is the usual Euclidean norm in $\mathbf{R}^{k}$ and $\lambda$ is the canonical Gaussian measure in $\mathbf{R}^{k}$.

(ii) Let $x^{*}$ be as in (3.6). For the rest of the proof, we set $\varphi=D \Phi\left(x^{*}\right)$. Since for any $\xi \in E^{*}$, $\sup _{\eta \in E^{*}}\left[\langle\eta, \Delta(\xi)\rangle-\frac{1}{2} \int \eta^{2} d \mu\right]$ is attained at $\eta=\xi$, as 
is easily proved, we have by Lemma $3.2(\mathrm{i})$

$$
I\left(x^{*}\right)=\left\langle\varphi, x^{*}\right\rangle-\frac{1}{2} \int \varphi^{2} d \mu .
$$

Therefore we may write

$$
\begin{aligned}
C_{n}= & \exp \left\{-\frac{b_{n}^{2}}{n}\left[\Phi\left(x^{*}\right)-I\left(x^{*}\right)\right]\right\} E \exp \left\{\frac{b_{n}^{2}}{n} \Phi\left(\frac{S_{n}}{b_{n}}\right)\right\} \\
= & \exp \left\{-\frac{b_{n}^{2}}{n} \frac{1}{2} \int \varphi^{2} d \mu\right\} E\left(\operatorname { e x p } \left\{\frac { b _ { n } ^ { 2 } } { n } \left[\Phi\left(x^{*}+\left(\frac{S_{n}}{b_{n}}-x^{*}\right)\right)\right.\right.\right. \\
& \left.\left.\left.-\Phi\left(x^{*}\right)-\left\langle\varphi, \frac{S_{n}}{b_{n}}-x^{*}\right\rangle\right]\right\} \exp \left\{\frac{b_{n}}{n}\left\langle\varphi, S_{n}\right\rangle\right\}\right) \\
=\left(\hat{\mu}\left(\frac{b_{n}}{n} \varphi\right)\right)^{n} \exp \left\{-\frac{b_{n}^{2}}{n} \frac{1}{2} \int \varphi^{2} d \mu\right\} E \exp \left\{\frac { b _ { n } ^ { 2 } } { n } \left[\Phi\left(x^{*}+u_{n}+\frac{T_{n}}{b_{n}}\right)\right.\right. & \left.\left.-\Phi\left(x^{*}\right)-\left\langle\varphi, u_{n}+\frac{T_{n}}{b_{n}}\right\rangle\right]\right\},
\end{aligned}
$$

where $T_{n}=\sum_{j=1}^{n}\left(Y_{n j}-E\left(Y_{n j}\right)\right)$, with $\left\{Y_{n j}: j=1, \ldots, n\right\}$ independent and

$$
d \mathscr{L}\left(Y_{n j}\right)=\frac{e^{b_{n} \varphi / n}}{\hat{\mu}\left(\frac{b_{n}}{n} \varphi\right)} d \mu, \quad \text { and } \quad u_{n}=\frac{n}{b_{n}} E\left(Y_{n 1}\right)-x^{*} .
$$

Now let $F_{n}(x)=\Phi\left(x^{*}+u_{n}+x\right)-\Phi\left(x^{*}\right)-\left\langle\varphi, u_{n}+x\right\rangle$. Since

$$
\exp \left\{\frac{b_{n}^{2}}{n} F_{n}\left(\frac{T_{n}}{b_{n}}\right)\right\}=f_{n}\left(T_{n} / n^{1 / 2}\right),
$$

where $f_{n}$ is as in Lemma 3.4 , in order to prove $\lim _{n \rightarrow \infty} C_{n}=C$ it is enough by Lemma 3.4 to prove

$$
\begin{aligned}
& \lim _{n \rightarrow \infty}\left(\hat{\mu}\left(\frac{b_{n}}{n} \varphi\right)\right)^{n} \exp \left\{-\frac{b_{n}^{2}}{n} \frac{1}{2} \int \varphi^{2} d \mu\right\}=1 . \\
& \left\{\exp \left\{\frac{b_{n}^{2}}{n} F_{n}\left(\frac{T_{n}}{b_{n}}\right)\right\}\right\} \text { is uniformly integrable. }
\end{aligned}
$$

We prove (a) first. A straightforward expansion gives

$$
\begin{aligned}
\hat{\mu}\left(\frac{b_{n}}{n} \varphi\right)= & +\frac{1}{2}\left(\frac{b_{n}}{n}\right)^{2} \int \varphi^{2} d \mu \\
& +\frac{1}{6}\left(\frac{b_{n}}{n}\right)^{3} \int \varphi^{3} d \mu+O\left(\left(\frac{b_{n}}{n}\right)^{4}\right) .
\end{aligned}
$$

On the other hand,

$$
\exp \left\{-\frac{1}{2} \frac{b_{n}^{2}}{n^{2}} \int \varphi^{2} d \mu\right\}=1-\frac{1}{2}\left(\frac{b_{n}}{n}\right)^{2} \int \varphi^{2} d \mu+O\left(\left(\frac{b_{n}}{n}\right)^{4}\right)
$$

Therefore

$$
\hat{\mu}\left(\frac{b_{n}}{n} \varphi\right) \exp \left\{-\frac{1}{2}\left(\frac{b_{n}}{n}\right)^{2} \int \varphi^{2} d \mu\right\}=1-\left(\frac{b_{n}}{n}\right)^{3} r_{n},
$$


where $\left\{r_{n}\right\}$ is bounded. From assumption (1.5) it follows that

$$
\left(\hat{\mu}\left(\frac{b_{n}}{n} \varphi\right)\right)^{n} \exp \left\{-\frac{1}{2} \frac{b_{n}^{2}}{n} \int \varphi^{2} d \mu\right\}=\left(1-\frac{\left(b_{n} / n^{2 / 3}\right)^{3} r_{n}}{n}\right)^{n} \rightarrow 1 .
$$

(It is clear from the argument that if $\int \varphi^{3} d \mu=0$, then the conclusion will hold under the weaker assumption $b_{n} / n^{3 / 4} \rightarrow 0$.)

We turn now to the proof of (b). In order to prove this assertion, it is clearly enough to prove:

(I) For every $\delta>0$,

$$
\limsup _{n \rightarrow \infty} E\left(\exp \left\{\frac{b_{n}^{2}}{n} F_{n}\left(\frac{T_{n}}{b_{n}}\right)\right\} I_{\left\{\left\|T_{n} / b_{n}\right\| \geq \delta\right\}}\right)=0 .
$$

(II) There exist $\delta>0, p>1$ such that

$$
\sup _{n} E\left(\exp \left\{p \frac{b_{n}^{2}}{n} F_{n}\left(T_{n} / b_{n}\right)\right\} I_{\left\{\left\|T_{n} / b_{n}\right\|<\delta\right\}}\right)<\infty .
$$

In order to prove (I), it suffices to show that for any closed set $D$,

$$
\limsup _{n \rightarrow \infty} \frac{n}{b_{n}^{2}} \log \int_{D} \exp \left\{\frac{b_{n}^{2}}{n} F_{n}\right\} d \lambda_{n} \leq \sup _{x \in D}[F(x)-I(x)],
$$

where $\lambda_{n}=\mathscr{L}\left(T_{n} / b_{n}\right)$ and $F(x)=\Phi\left(x^{*}+x\right)-\Phi\left(x^{*}\right)-\langle\varphi, x\rangle$. Indeed, a simple computation using Lemma 3.2(i) gives

$$
F(x)-I(x)=\left[\Phi\left(x^{*}+x\right)-I\left(x^{*}+x\right)\right]-\left[\Phi\left(x^{*}\right)-I\left(x^{*}\right)\right] .
$$

Now let $D=\{x \in E:\|x\| \geq \delta\}$. Arguing as in the proof of Lemma 3.2(i) one can see that $s=\sup _{x \in D}[F(x)-I(x)]$ is attained, and assumption (3.6) together with (3.28) imply that $s<0$. Thus (3.27) implies (I).

To establish (3.27) we first show that

$$
\lim _{t \rightarrow \infty} \limsup _{n \rightarrow \infty} \frac{n}{b_{n}^{2}} \log \int_{\left\{F_{n} \geq t\right\}} \exp \left\{\frac{b_{n}^{2}}{n} F_{n}\right\} d \lambda_{n}=-\infty .
$$

By assumption (3.5) and Lemma 3.4, there exist $\alpha, \beta>0$ such that $\sup _{n} F_{n}(x)$ $\leq \alpha\|x\|+\beta$ for all $x \in E$. It follows that

$$
\begin{aligned}
\int_{\left\{F_{n} \geq t\right\}} \exp \left\{b_{n}^{2} F_{n}\right\} d \lambda_{n} & \leq \exp \left\{-\frac{b_{n}^{2}}{n} t\right\} \int \exp \left\{2 \frac{b_{n}^{2}}{n} F_{n}\right\} d \lambda_{n} \\
& \leq \exp \left\{-\frac{b_{n}^{2}}{n} t\right\} E \exp \left\{2 \frac{b_{n}^{2}}{n}\left(\alpha\left\|\frac{T_{n}}{b_{n}}\right\|+\beta\right)\right\} .
\end{aligned}
$$

By the argument in Lemma 2.1(i) (with $q_{K}$ replaced by $\|\cdot\|$ ), the second factor is bounded by $\exp \left\{b_{n}^{2} d / n\right\}$ for some constant $d>0$ and sufficiently large $n$. Therefore

$$
\limsup _{n \rightarrow \infty} \frac{n}{b_{n}^{2}} \log \int_{\left\{F_{n} \geq t\right\}} \exp \left\{\frac{b_{n}^{2}}{n} F_{n}\right\} d \lambda_{n} \leq-(t-d),
$$

which yields (3.29). We proceed now to complete the proof of (3.27). Let $a_{n}=b_{n}^{2} / n$ and fix $t>0$. By the argument of the proof of Theorem 2.2(i), given $\tau>0$ there exist a compact set $K_{\tau}$ and $n_{0} \in \mathbf{N}$ such that for $n \geq n_{0}$,

$$
P\left\{T_{n} / b_{n} \in K_{\tau}^{c}\right\} \leq e^{-a_{n} \tau} .
$$


By Lemma 3.4, $F_{n} \rightarrow F$ uniformly over compact sets. Therefore given $\delta>0$, we have for sufficiently large $n$

$$
\begin{aligned}
\int_{D} \exp \left\{a_{n} \min \left(F_{n}, t\right)\right\} d \lambda_{n} \\
\leq \int_{D \cap K_{\tau}} \exp \left\{a_{n} \min \left(F_{n}, t\right)\right\} d \lambda_{n}+\int_{K_{\tau}^{c}} \exp \left\{a_{n} t\right\} d \lambda_{n} \\
\leq \int_{D \cap K_{\tau}} \exp \left\{a_{n} \min (F+\delta, t)\right\} d \lambda_{n}+e^{-a_{n}(\tau-t)} .
\end{aligned}
$$

By [15, p. 321], taking into account Theorem 2.2(i)

$$
\begin{aligned}
\limsup _{n \rightarrow \infty} & a_{n}^{-1} \log \int_{D \cap K_{\tau}} \exp \left\{a_{n} \min (F+\delta, t)\right\} d \lambda_{n} \\
& \leq \sup _{x \in D \cap K_{\tau}}[\min (F(x)+\delta, t)-I(x)] \\
& \leq \delta+\sup _{x \in D}[F(x)-I(x)]
\end{aligned}
$$

and it follows that

$$
\limsup _{n \rightarrow \infty} a_{n}^{-1} \log \int_{D} \exp \left\{a_{n} \min \left(F_{n}, t\right)\right\} d \lambda_{n} \leq \sup _{x \in D}[F(x)-I(x)] .
$$

Now a standard argument using (3.29) completes the proof of (3.27), and hence that of $(\mathrm{I})$.

We turn now to the proof of (II). By Taylor's formula,

$$
F_{n}\left(\frac{T_{n}}{b_{n}}\right)=\frac{1}{2} D^{2} \Phi\left(x^{*}+\theta\left(u_{n}+\frac{T_{n}}{b_{n}}\right)\right)\left(u_{n}+\frac{T_{n}}{b_{n}}, u_{n}+\frac{T_{n}}{b_{n}}\right),
$$

where $|\theta|<1$, and therefore

$$
\frac{b_{n}^{2}}{n} F_{n}\left(\frac{T_{n}}{b_{n}}\right)=\frac{1}{2} D^{2} \Phi\left(x^{*}+\theta\left(u_{n}+\frac{T_{n}}{b_{n}}\right)\right)\left(\frac{b_{n}}{n^{1 / 2}} u_{n}+\frac{T_{n}}{n^{1 / 2}}, \frac{b_{n}}{n^{1 / 2}} u_{n}+\frac{T_{n}}{n^{1 / 2}}\right) .
$$

By the assumptions on $\Phi$ and Lemma 3.4, simple estimates show that given $\varepsilon>0$ (to be further specified later), it is possible to choose $\delta_{1}>0$ and $n_{1} \in \mathbf{N}$ such that for $n \geq n_{1}$,

$$
\text { (3.30) } \frac{b_{n}^{2}}{n} F_{n}\left(\frac{T_{n}}{b_{n}}\right) \leq \varepsilon+\varepsilon\left\|\frac{T_{n}}{n^{1 / 2}}\right\|^{2}+\frac{1}{2} A\left(\frac{T_{n}}{n^{1 / 2}}, \frac{T_{n}}{n^{1 / 2}}\right) \text { on }\left\{\left\|\frac{T_{n}}{b_{n}}\right\|<\delta_{1}\right\} \text {, }
$$

where $A=D^{2} \Phi\left(x^{*}\right)$. Let $p>1, r>1$ (to be further specified later), and define $s>1$ by $r^{-1}+s^{-1}=1$. Let $\left\{\xi_{j}: j \in \mathbf{N}\right\} \subset E^{*}$ be such that $\left\{\Delta\left(\xi_{j}\right): j \in \mathbf{N}\right\}$ is an orthonormal basis of $H_{\mu}$. By Lemma 3.3 and Hölder's inequality, for any $\delta \leq \delta_{1}, n \geq n_{1}$ and $k \in \mathbf{N}$, we have

$$
\begin{aligned}
(3.31) E\left(\exp \left\{p\left(\varepsilon\left\|\frac{T_{n}}{n^{1 / 2}}\right\|^{2}+\frac{1}{2} A\left(\frac{T_{n}}{n^{1 / 2}}, \frac{T_{n}}{n^{1 / 2}}\right)\right)\right\} I_{\left\{\left\|T_{n} / b_{n}\right\|<\delta\right\}}\right) \\
\leq\left(E\left(\exp \left\{s p\left(\varepsilon\left\|\frac{T_{n}}{n^{1 / 2}}\right\|^{2}+\frac{d}{2}\left(1+\beta^{2}\right)\left\|Q_{k}\left(\frac{T_{n}}{n^{1 / 2}}\right)\right\|^{2}\right)\right\} I_{\left\{\left\|T_{n} / b_{n}\right\|<\delta\right\}}\right)\right)^{1 / s} \\
\cdot\left(E\left(\exp \left\{r \frac{p}{2}\left(\alpha+\beta^{-2} d \sigma^{2}\right) \sum_{j=1}^{k}\left\langle\xi_{j}, \frac{T_{n}}{n^{1 / 2}}\right\rangle^{2}\right\} I_{\left\{\left\|T_{n} / b_{n}\right\|<\delta\right\}}\right)\right)^{1 / r}
\end{aligned}
$$


Since $\alpha<1$, we may choose and fix $\beta>0, p>1, r>1$ in such a way that $r p\left(\alpha+\beta^{-2} d \sigma^{2}\right)<1$. For $x \in E$, let

$$
q_{k}(x)=\varepsilon^{1 / 2}\|x\|+\left(\frac{d}{2}\left(1+\beta^{2}\right)\right)^{1 / 2}\left\|Q_{k}(x)\right\|,
$$

and let $c_{k}$ be such that $q_{k} \leq c_{k}\|\cdot\|$. Then the $s$ th power of the first factor in the right-hand side of (3.31) is bounded by

$$
E\left(\exp \left\{s p q_{k}^{2}\left(T_{n} / n^{1 / 2}\right)\right\} I_{\left\{q_{k}\left(T_{n}\right)<\delta c_{k} n\right\}}\right)
$$

for sufficiently large $n$, since $b_{n} / n \rightarrow 0$. By [12], one may choose $k \in \mathbf{N}$, $\varepsilon>0$ such that

$$
\int q_{k}^{2} d \gamma<(8 s p)^{-1}
$$

Since $\left\{\mu^{* n}\left(n^{1 / 2}(\cdot)\right)\right\}$ converges weakly to $\gamma$, by the results of [4] one may choose $h \in \mathbf{N}$ such that

$$
\int q_{k}^{2} d \mu^{* h}\left(h^{1 / 2}(\cdot)\right)<(8 s p)^{-1}
$$

and since $\left\{\mathscr{L}\left(Y_{n 1}-E Y_{n 1}\right)\right\}$ converges weakly to $\mu$, we have by [4]

$$
\limsup _{n \rightarrow \infty} E q_{k}^{2}\left(h^{-1 / 2} \sum_{j=1}^{h}\left(Y_{n j}-E Y_{n j}\right)\right)<(8 s p)^{-1} \text {. }
$$

The triangular array $\left\{Y_{n j}-E Y_{n j}: n \in \mathbf{N}, j=1, \ldots, n\right\}$ and the seminorm $q_{k}$ satisfy the assumptions of Lemma 3.5 (assumption (3) follows from Lemma 3.4 and [4]). Therefore for sufficiently small $\delta>0$,

$$
\sup _{n} E\left(\exp \left\{s p q_{k}^{2}\left(T_{n} / n^{1 / 2}\right)\right\} I_{\left\{q_{k}\left(T_{n}\right)<\delta c_{k} n\right\}}\right)<\infty .
$$

Finally, by Lemma 3.6, it is possible to choose $\delta>0$ such that the second factor in the right-hand side of (3.31) is bounded uniformly in $n$. Now assertion (II) follows from (3.30), (3.31) and (3.33).

\section{REFERENCES}

1. A. de Acosta, Upper bounds for large deviations of dependent random vectors, Z. Wahrsch. Verw. Gebiete 69 (1985), 551-565.

2. __ On large deviations of sums of independent random vectors, Lecture Notes in Math., vol. 1153, Springer-Verlag, Berlin and New York, 1985, pp. 1-14.

3. A. de Acosta, A. Araujo, and E. Giné, On Poisson measures, Gaussian measures and the central limit theorem in Banach spaces, Advances in Probability, vol. IV, Dekker, New York, 1978, pp. 1-68.

4. A. de Acosta and E. Giné, Convergence of moments and related functionals in the general central limit theorem in Banach spaces, Z. Wahrsch. Verw. Gebiete 48 (1979), 213-231.

5. A. de Acosta and J. Kuelbs, Some results on the cluster set $C\left(\left\{S_{n} / a_{n}\right\}\right)$ and the LIL, Ann. Probab. 11 (1983), 102-122.

6. A. Araujo and E. Giné, The central limit theorem for real and Banach valued random variables, Wiley, New York, 1980.

7. R. Azencott, Grandes déviations et applications, Lecture Notes in Math., vol. 774, SpringerVerlag, Berlin and New York, 1980.

8. R. R. Bahadur and S. Zabell, Large deviations of the sample mean in general vector spaces, Ann. Probab. 7 (1979), 587-621.

9. P. Billingsley, Convergence of probability measures, Wiley, New York, 1968. 
10. E. Bolthausen, Laplace approximations for sums of independent random vectors. Probab. Theory Related Fields 72 (1986), 305-318.

11. _ Laplace approximations for sums of independent random vectors. Part II, Probab. Theory Related Fields 76 (1987), 167-206.

12. C. Borell, Gaussian Radon measures on locally convex spaces, Math. Scand. 38 (1976), 265-284.

13. A. A. Borovkov and A. A. Mogulskii, Probabilities of large deviations in topological spaces. I, Siberian Math. J. 19 (1978), 697-709.

14. M. D. Donsker and S. R. S. Varadhan, Asymptotic evaluation of certain Markov process expectations for large time. III, Comm. Pure Appl. Math. 29 (1976), 389-461.

15. R. Ellis, Entropy, large deviations and statistical mechanics. Springer-Verlag, Berlin and New York, 1985.

16. W. Feller, An introduction to probability theory and its applications, vol. 2, Wiley, New York, 1971.

17. M. I. Freidlin and A. D. Wentzell, Random perturbations of dynamical systems, SpringerVerlag, Berlin and New York, 1984.

18. A. Martin-Löf, Laplace approximation for sums of independent random variables, $\mathrm{Z}$. Wahrsch. Verw. Gebiete 59 (1982), 101-115.

19. V. V. Petrov, Sums of independent random variables, Springer-Verlag, Berlin and New York, 1975.

20. S. R. S. Varadhan, Large deviations and applications, SIAM, Philadelphia, Pa., 1984.

21. V. V. Yurinskii, Exponential inequalities for sums of random vectors, J. Multivariate Anal. 6 (1976), 473-499.

Department of Mathematics and Statistics, Case Western Reserve University, CleveLAND, OHIO 44106 